\title{
MULTIPLE KÜNNETH FORMULAS FOR ABELIAN GROUPS
}

\author{
BY
}

THOMAS W. HUNGERFORD

1. Introduction. Bockstein [6], Cartan and Eilenberg [2], Kelly [7], Dold [8] and MacLane [5], among others, have proved the Künneth Theorem for two chain complexes of abelian groups under various hypotheses. MacLane [4] proves an analogous theorem for three complexes and Bockstein [1], [9] states and proves an analogue for $n$ complexes (under unnecessarily strong hypotheses, as it turns out). The purpose of this paper is to prove, under relatively weak hypotheses, a multiple Künneth formula for $\boldsymbol{n}$ chain complexes of abelian groups ( $n \geqq 2$ ) which subsumes and, in part, extends all of these previous results.

If $K^{1}$ and $K^{2}$ are (not necessarily positive) chain complexes of abelian groups such that $H\left(\operatorname{Tor}\left(K^{1}, K^{2}\right)\right)=0$, then the Künneth Theorem states that there is a split exact sequence:

(1) $0 \rightarrow H\left(K^{1}\right) \otimes H\left(K^{2}\right) \stackrel{\alpha}{\longrightarrow} H\left(K^{1} \otimes K^{2}\right) \stackrel{\beta}{\longleftarrow} \underset{\gamma}{\longleftrightarrow}$ Tor $\left(H\left(K^{1}\right), H\left(K^{2}\right)\right) \rightarrow 0$

in which the maps $\alpha$ and $\beta$ are natural, but the splitting map $\gamma$ is not. Thus the homology of $K^{1} \otimes K^{2}$ is the direct sum of the (left) derived functors of $H\left(K^{1}\right) \otimes H\left(K^{2}\right)$. A proof is given in Dold [8] and Kelly [7]; Cartan and Eilenberg [2] obtain the sequence, but not the splitting; MacLane [5] obtains the split sequence under the stronger assumption that one of the complexes is torsionfree.

In MacLane [4] the well-known description of the functor Tor $(A, B)$ by generators and relations is extended to define a functor Tor $(A, B, C)$ of three variables. Another functor Trip $(A, B, C)$ is defined as a certain quotient of the direct sum $\operatorname{Tor}(A, B) \otimes C \oplus \operatorname{Tor}(B, C) \otimes A \oplus \operatorname{Tor}(A, C) \otimes B$ and the following theorem is proved.

TRIPLE KüNNETH TheOREM. If $K^{1}, K^{2}$, and $K^{3}$ are complexes of abelian groups, two of which are torsion-free, then then is a chain of subgroups: $P_{0} \subset P_{1} \subset P_{2}=H\left(K^{1} \otimes K^{2} \otimes K^{3}\right)$ for which there are natural isomorphisms of graded groups:

Presented the Society, January 27, 1965; received by the editors February 7, 1964 and, in revised form, February 27, 1964. 


$$
\begin{aligned}
H\left(K^{1}\right) \otimes H\left(K^{2}\right) \otimes H\left(K^{3}\right) & \cong P_{0} ; \\
\operatorname{Trip}\left(H\left(K^{1}\right), H\left(K^{2}\right), H\left(K^{3}\right)\right) & \cong P_{1} / P_{0} ; \\
\operatorname{Tor}\left(H\left(K^{1}\right), H\left(K^{2}\right), H\left(K^{3}\right)\right) & \cong P_{2} / P_{1} .
\end{aligned}
$$

It is also shown that Trip and Tor are in fact the first and second (left) derived functors of the triple tensor product $A \otimes B \otimes C$. Although it is not proved there, it is in fact true that $H\left(K^{1} \otimes K^{2} \otimes K^{3}\right) \cong P_{0} \oplus P_{1} / P_{0} \oplus P_{2} / P_{1}$, i.e., the homology of $K^{1} \otimes K^{2} \otimes K^{3}$ is the direct sum of the (left) derived functors of

$$
H\left(K^{1}\right) \otimes H\left(K^{2}\right) \otimes H\left(K^{3}\right) .
$$

Bockstein ([1], [9]) has extended these concepts by defining inductively for each $k \geqq 2$ groups $\operatorname{Tor}\left(A^{1}, \cdots, A^{k}\right)$ and for each $i(1 \leqq i \leqq n)$ groups $\operatorname{Mult}_{i}\left(A^{1}, \cdots, A^{n}\right)$ (which is a certain quotient of the direct sum

$$
\sum \operatorname{Tor}\left(A^{s_{1}}, \cdots, A^{s^{i}}\right) \otimes A^{r_{1}} \otimes \cdots \otimes A^{r_{n-1}},
$$

the sum being taken over all permutations $\left\{s_{1}, \cdots, s_{i}, r_{1}, \cdots, r_{n-i}\right\}$ of $1, \cdots, n$ for which $s_{1}<s_{2}<\cdots<s_{i}$ and $\left.r_{1}<r_{2}<\cdots<r_{n-i}\right)$. It is stated and proved that for $n$ torsion-free complexes $K_{1}, \cdots, K_{n}$,

$$
H\left(K^{1} \otimes \cdots \otimes K^{n}\right)=\sum_{i=1}^{n} \operatorname{Mult}_{i}\left(H\left(K^{1}\right), \cdots, H\left(K^{n}\right)\right) .
$$

However, Bockstein's statement that the theorem is not true if only $n-1$ of the complexes is torsion-free is incorrect. [This statement is based essentially on an example in the case $n=2$; but the example is false (a minus sign is omitted in computing the boundary of $K \otimes L)$.] As we shall show below an even stronger conclusion holds under considerably weaker hypotheses; furthermore we shall show that the functors $\operatorname{Mult}_{i}\left(A^{1}, \cdots, A^{n}\right)$ are (after a change of indices) precisely the (left) derived functors of the $n$-fold tensor product $A^{1} \otimes \cdots \otimes A^{n}$.

Hereafter we shall use the following terminology. For any given $n \geqq 2$ we define the functor $\operatorname{Mult}_{i}^{n}\left(A^{1}, \cdots, A^{n}\right)$ to be the $i$ th (left) derived functor of the $n$-fold tensor product $A^{1} \otimes \cdots \otimes A^{n}$, i.e.,

$$
\operatorname{Mult}_{i}^{n}\left(A^{1}, \cdots, A^{n}\right)=H_{i}\left(K^{1} \otimes \cdots \otimes K^{n}\right),
$$

where for each $r, K^{r}$ is a free resolution of $A^{r}$. In situations where no confusion can arise we shall often write Mult $_{i}$ in place of Mult ${ }_{i}^{n}$.

The most general approach to the question is given by Cartan and Eilenberg [2] (and also in MacLane [5]). For any complexes $K^{1}, \cdots, K^{n}$ they define the so-called hyperhomology group $\mathscr{L}\left(K^{1} \otimes \cdots \otimes K^{n}\right)$ to be the graded group $H\left(R^{1} \otimes \cdots \otimes R^{n}\right)$, where each $R^{r}$ is a double complex projective resolution (in their sense) of the complex $K^{r}(r=1, \cdots, n)$ and the homology group is taken relative to the total differential in $R^{1} \otimes \cdots \otimes R^{n}$. Furthermore they show that there 
are two spectral sequences (denoted I and II) both converging to $\mathscr{L}\left(K^{1} \otimes \cdots \otimes K^{\prime \prime}\right)$, and having the initial terms:

$$
\begin{aligned}
\mathrm{I}_{p, q}^{2} & =H_{p}\left(\operatorname{Mult}_{q}^{n}\left(K^{1}, \cdots, K^{n}\right)\right), \\
\mathrm{II}_{p, q}^{2} & =\sum \operatorname{Mult}_{q}^{n}\left(H_{p 1}\left(K^{1}\right), \cdots, H_{p_{n}}\left(K^{n}\right)\right),
\end{aligned}
$$

where the sum is taken over all $n$-tuples $\left(p_{1}, \cdots, p_{n}\right)$ such that $\sum_{r=1}^{n} p_{r}=p$.

In the case $n=2$ [and hence Mult ${ }^{n}=$ Tor] there is, for any two complexes of abelian groups $K^{1}$ and $K^{2}$, an exact sequence:

$$
0 \rightarrow H\left(K^{1}\right) \otimes H\left(K^{2}\right) \rightarrow \mathscr{L}\left(K^{1} \otimes K^{2}\right) \rightarrow \operatorname{Tor}\left(H\left(K^{1}\right), H\left(K^{2}\right)\right) \rightarrow 0 .
$$

It is shown that if $H\left(\operatorname{Tor}\left(K^{1}, K^{2}\right)\right)=0$, then $\mathscr{L}\left(K^{1} \otimes K^{2}\right)$ is naturally isomorphic to $H\left(K^{1} \otimes K^{2}\right)$ and the sequence (2) reduces to the usual Künneth sequence (1). No statement is made about either of the sequences (1) or (2) splitting.

In this paper we shall prove the following multiple Künneth formula, which generalizes the previous results for complexes of abelian groups and also shows the relationship between the hyperhomology group and the derived functors of the $n$-fold tensor product.

THEOREM 1.1. If $K^{1}, \cdots, K^{n}$ are chain complexes of abelian groups, then there is an isomorphism of graded groups:

$$
\mathscr{L}\left(K^{1} \otimes \cdots \otimes K^{n}\right) \cong \sum_{i=0}^{n-1} \operatorname{Mult}_{i}^{i i}\left(H\left(K^{1}\right), \cdots, H\left(K^{n}\right)\right) ;
$$

in particular,

$$
\mathscr{L}_{k}\left(K^{1}|\otimes \cdots \otimes| K^{n}\right) \cong \sum_{i=0}^{n-1} \sum \operatorname{Mult}_{i}^{n}\left(H_{p_{1}}\left(K^{1}\right), \cdots, H_{p_{n}}\left(K^{n}\right)\right),
$$

where the second sum is over all $n$-tuples $\left(p_{1}, \cdots, p_{n}\right)$ such that $\overleftarrow{\Sigma}_{r=1}^{n} p_{r}=k-i$. The isomorphism is not natural; however, it induces natural maps

$$
f_{0}: \operatorname{Mult}_{0}^{n}\left(H\left(K^{1}\right), \cdots, H\left(K^{n}\right)\right) \rightarrow \mathscr{L}\left(K^{1} \otimes \cdots \otimes K^{n}\right),
$$

and for $0<i \leqq n-1$,

$$
f_{i}: \operatorname{Mult}_{i}^{n}\left(H\left(K^{1}\right), \cdots, H\left(K^{n}\right)\right) \rightarrow \text { Cokernel } f_{i-1} .
$$

COROLlARY 1.2. If $K^{1}, \cdots, K^{n}$ are chain complexes of abelian groups such that

$$
H\left(\operatorname{Mult}_{i}^{n}\left(K^{1}, \cdots, K^{n}\right)\right)=0 \text { for all } 0<i \leqq n-1,
$$

then Theorem 1.1 remains true if $\mathscr{L}\left(K^{1} \otimes \cdots \otimes K^{n}\right)$ is replaced by $H\left(K^{1} \otimes \cdots \otimes K^{n}\right)$.

COROLlaRY 1.3. If $K^{1}, \cdots, K^{n}$ are chain complexes of abelian groups, then the hyperhomology group $\mathscr{L}\left(K^{1} \otimes \cdots \otimes K^{n}\right)$ is completely determined by $H\left(K^{1}\right), \cdots, H\left(K^{n}\right)$. 
Corollary 1.3 follows immediately from the theorem. Under the hypotheses of Corollary 1.2 the spectral sequence I associated with $\mathscr{L}\left(K^{1} \otimes \cdots \otimes K^{n}\right)$ collapses and thus there is a natural isomorphism (for each $k$ )

$$
\mathrm{I}_{k, 0}^{2}=H\left(K^{1} \otimes \cdots \otimes K^{n}\right) \cong \mathscr{L}_{k}\left(K^{1} \otimes \cdots \otimes K^{n}\right) .
$$

It should be noted that if $n-1$ of the complexes $K^{1}, \cdots, K^{n}$ are torsion-free, then

$$
\operatorname{Mult}_{i}^{n}\left(K^{1}, \cdots, K^{n}\right)=0 \text { for all } i>0 \text {; }
$$

thus the hypotheses of Corollary 1.2 are fulfilled in this case. For $n=2$ this states the obvious fact that $\operatorname{Tor}\left(K^{1}, K^{2}\right)=0$ if one of $K^{1}, K^{2}$ is torsion-free. For larger $n$ the statement follows by (double) induction on $i$ and $n$ from the exact sequence:

$$
\begin{aligned}
0 \rightarrow K^{1} \otimes \operatorname{Mult}_{i}^{n-1}\left(K^{2}, \cdots, K^{n}\right) & \rightarrow \operatorname{Mult}_{i}^{n}\left(K^{1}, \cdots, K^{n}\right) \\
& \rightarrow \operatorname{Tor}\left(K^{1}, \operatorname{Mult}_{i-1}^{n-1}\left(K^{2}, \cdots, K^{n}\right)\right) \rightarrow 0 .
\end{aligned}
$$

This is just the Künneth sequence (1) applied to the complexes $R^{1}$ and $\left(\bar{K}^{2} \otimes \cdots \otimes \bar{K}^{n}\right)$, where each $\bar{K}^{r}$ is a free resolution (in the usual sense) of $K^{r}(r=1, \cdots, n)$.

Outline of the proof of Theorem 1.1. For each $r(r=1,2, \cdots, n)$ let $R^{r}$ be a double complex projective resolution of $K^{r}$, as defined in Chapter XVII of [2]: denote the two boundary operators in $K^{r}$ by $d_{1}^{r}$ and $d_{2}^{r}$. Then it follows from the definition of $K^{r}$ that $\bar{R}^{r}$ is free and $H\left(R^{r}\right) \cong H\left(K^{r}\right)$ (naturally). Also, by definition,

$$
\mathscr{L}\left(K^{1} \otimes \cdots \otimes K^{n}\right)=H\left(R^{1} \otimes \cdots \otimes K^{n}\right),
$$

where the boundary in $\bar{K}^{1} \otimes \cdots \otimes K^{n}$ is given by

$$
\partial=\partial_{1}+\partial_{2}
$$

where

$$
\begin{aligned}
& \partial_{1}=\sum_{r=1}^{n}(-1)^{*} 1 \otimes \cdots \otimes 1 \otimes d_{1}^{r} \otimes 1 \otimes \cdots \otimes 1 ; \\
& \partial_{2}=\sum_{r=1}^{n}(-1)^{*} 1 \otimes \cdots \otimes 1 \otimes d_{2}^{r} \otimes 1 \otimes \cdots \otimes 1 ;
\end{aligned}
$$

and $(-1)^{*}$ is the usual sign determined by the standard sign convention, as given by Cartan and Eilenberg [2, Chapter IV, §5]. If we consider each $R^{r}$ as a single complex with boundary $d_{1}^{r}+d_{2}^{r}$, then $R^{1} \otimes \cdots \otimes R^{n}$ is a complex in the usual way with boundary

$$
\sum_{r=1}^{n}(-1)^{*} 1 \otimes \cdots \otimes 1 \otimes\left(d_{1}^{r}+d_{2}^{r}\right) \otimes 1 \otimes \cdots \otimes 1 .
$$

This is the same "total boundary" as (3). 
It follows that in order to prove the theorem it will suffice to show that for any torsion-free complexes $K^{1}, \cdots, K^{n}$, there is an isomorphism of graded groups:

$$
H\left(K^{1} \otimes \cdots \otimes K^{n}\right) \cong \sum_{i=0}^{n-1} \operatorname{Mult}_{i}^{n}\left(H\left(K^{1}\right), \cdots, H\left(K^{n}\right)\right) .
$$

Fo if there is such an isomorphism and we apply it in the case of $R^{1}, \cdots, \bar{K}^{n}$ (as above) then we obtain Theorem 1.1.

We now proceed to prove the existence of the isomorphism (4). $\S \S 2-4$ are devoted to developing machinery for this purpose. Although the basic idea underlying the proof given in Bockstein [9] is substantially the same as the idea used here, his procedure and technique differ somewhat and his final result is considerably weaker.

2. Definitions and notation. We assume that the definition of a Bockstein spectrum and any necessary properties of spectra are known (see [3]) $\left({ }^{1}\right)$. An important example of a Bockstein spectrum is the so-called homology spectrum of a (not necessarily positive) chain complex $K$ of torsion-free abelian groups. It is denoted $\{H(K, m)\}$ and consists of the groups $H(K)=H(K, 0)$ and $H\left(K \otimes Z_{m}\right)=H(K, m)$ (for all $\left.m>0\right)$, together with the coefficient homomorphisms (for each $m, k \geqq 0$ )

$$
\begin{array}{cc}
\lambda_{m}^{m k}: H(K, m k) \rightarrow H(K, m) & (m k \geqq 0), \\
\mu_{m k}^{m}: H(K, m) \rightarrow H(K, m k) & (m k>0)
\end{array}
$$

induced by the canonical maps $Z_{m k} \rightarrow Z_{m}$ and $Z_{m} \rightarrow Z_{m k}$ (with $Z_{0}=Z$ ), and the Bockstein connecting homomorphism $\mu_{0}^{m}: H(K, m) \rightarrow H(K)(m>0)$ induced by the exact sequence $0 \rightarrow Z \stackrel{m}{\rightarrow} Z \rightarrow Z_{m} \rightarrow 0$. Note that $\mu_{0}^{m}$ has degree -1 and that all other $\lambda$ 's and $\mu$ 's have degree 0 .

DEFINITION 2.1. If $\left(B^{r},{ }^{r} \lambda,{ }^{r} \mu\right)(r=1,2, \cdots, n)$ are Bockstein spectra, then their tensor product $B^{1} \otimes \cdots \otimes B^{n}$ is the group $\left[\Sigma_{m \geqq 0} B_{m}^{1} \otimes \cdots \otimes B_{m}^{n}\right] / S$, where $S$ is the subgroup generated by all elements of the following forms [where $x^{i} \in B_{m}^{i}, y^{i} \in B_{m k}^{i}$, and $(-1)^{*}$ is the sign determined by the standard sign convention (as given by Cartan and Eilenberg [2, Chapter IV, §5])]:

$$
\begin{aligned}
& (-1)^{* 1} \lambda_{m}^{m k} y^{1} \otimes \cdots \otimes{ }^{r-1} \lambda_{m}^{m k} y^{r-1} \otimes x^{r} \otimes{ }^{r+1} \lambda_{m}^{m k} y^{r+1} \otimes \cdots \otimes{ }^{n} \lambda_{m}^{m k} y^{n} \\
& -(-1)^{*} y^{1} \otimes \cdots \otimes y^{r-1} \otimes \mu_{m k}^{m} x^{r} \otimes y^{r+1} \otimes \cdots \otimes y^{n}(\text { for each } r=1,2, \cdots, n)
\end{aligned}
$$

$$
\sum_{r=1}^{n}(-1)^{*} x^{1} \otimes \cdots \otimes x^{r-1} \otimes r \lambda_{m}^{0} \mu_{0}^{m} x^{r} \otimes x^{r+1} \otimes \cdots \otimes x^{n}
$$

In the sequel we shall often denote the tensor product $B^{1} \otimes \cdots \otimes B^{n}$ (of groups, complexes, or spectra) by $\bigotimes_{i=1}^{n} B^{i}$.

(1) The concept of Bockstein spectrum (with somewhat different terminology) was first used by Bockstein and by J. H. C. Whitehead [10]. 
If $u$ is a cycle of a complex $K,|u|$ denotes its degree and $\eta(u)$ denotes its homology class in $H(K)$. If $\partial u=m \bar{u}$, then $\eta\left(u \otimes 1_{m}\right)$ denotes the homology class of the $m$-cycle $u \otimes 1_{m}$ in $H(K, m)$. If $K^{1}, \cdots, K^{n}$ are chain complexes of torsion-free abelian groups, we define a grading on the spectra tensor product $\bigotimes_{i=1}^{n}\left\{H\left(K^{i}, m\right)\right\}$ by defining the degree (of the class of) a generator $\eta\left(u^{1} \otimes 1_{m}\right) \otimes \cdots \otimes \eta\left(u^{n} \otimes 1_{m}\right)\left(u^{i} \in K^{i} ; \partial u^{i}=m \bar{u}^{i}\right)$ of $\bigotimes_{i=1}^{n} H\left(K^{i}, m\right)$ to be $\left|u^{1}\right|+\left|u^{2}\right|+\cdots+\left|u^{n}\right|-1$ if $m>0$ and $\left|u^{1}\right|+\cdots+\left|u^{n}\right|$ if $m=0$. (Note that this is consistent with the relations imposed by Definition 2.1 on the igroup $\left.\Sigma_{m \geqq 0}\left[\bigotimes_{i=1}^{n} H\left(K^{i}, m\right)\right].\right)$

3. The isomorphism theorem. The first step in the proof of Theorem 1.1 is to express $H\left(\bigotimes_{i=1}^{n} K^{i}\right)$ as a tensor product of Bockstein spectra as follows.

THEOREM 3.1. If $K^{1}, \cdots, K^{n}$ are chain complexes of torsion-free abelian groups, then there is an isomorphism of graded groups:

$$
\bigotimes_{i=1}^{n}\left\{H\left(K^{i}, m\right)\right\} \cong H\left(\bigotimes_{i=1}^{n} K^{i}\right) \text {. }
$$

Proof. For each $m \geqq 0$ define a map

$$
F(m): \bigotimes_{i=1}^{n} H\left(K^{i}, m\right) \longrightarrow H\left(\bigotimes_{i=1}^{n} K^{i}\right)
$$

as follows. If $m=0$, then

$$
F(0)=\alpha: \bigotimes_{i=1}^{n} H\left(K^{i}\right) \longrightarrow H\left(\bigotimes_{i=1}^{n} K^{i}\right),
$$

where $\alpha$ is the homology product map given by

$$
\alpha\left[\eta\left(u^{1}\right) \otimes \cdots \otimes \eta\left(u^{n}\right)\right]=\eta\left(u^{1} \otimes \cdots \otimes u^{n}\right) .
$$

If $m>0$, then $F(m)$ is the composition:

$$
\bigotimes_{i=1}^{n} H\left(K^{i}, m\right) \stackrel{\alpha}{\longrightarrow} H\left(K^{1} \otimes \cdots \otimes K^{n}, m\right) \stackrel{\delta_{0}^{m}}{\longrightarrow} H\left(\bigotimes_{i=1}^{n} K^{i}\right),
$$

where $\alpha$ is the homology product $(\bmod m)$ and $\delta_{0}^{m}$ is the Bockstein connecting homomorphism induced by the exact sequence $0 \rightarrow Z \stackrel{m}{\rightarrow} Z \rightarrow Z_{m} \rightarrow 0$. Hence the $F(m)$ define a map

$$
F: \sum_{m \geqq 0}\left[\bigotimes_{i=1}^{n} H\left(K^{i}, m\right)\right] \longrightarrow H\left(\bigotimes_{i=1}^{n} K^{i}\right) .
$$

A direct calculation (keeping in mind that the maps $\mu_{0}^{m}$ have degree -1 and all other maps have degree 0 ) shows that $F(S)=0$, where $S$ is the subgroup of 
Definition 2.1. Hence $F$ induces a well-defined homomorphism $F$ on the quotient $\Sigma_{m \geqq 0}\left[\bigotimes_{i=1}^{n} H\left(K^{i}, m\right)\right] / S$, i.e.,

$$
F: \bigotimes_{i=1}^{n}\left\{H\left(K^{i}, m\right)\right\} \longrightarrow H\left(\bigotimes_{i=1}^{n} K^{i}\right) .
$$

Since $F(m)$ has degree 0 for $m=0$ and degree -1 for $m>0, F$ is a map of graded groups. To show that $F$ is an isomorphism, it suffices to consider the case in which $K^{1}, \cdots, K^{n}$ are elementary complexes. In fact, the lemmas in the Appendix of [3] (extended to arbitrary $n$ in the obvious way) show that we need only consider the case in which $K^{r}(r=1, \cdots, n)$ is the complex (in dimensions 1 and 0$)$ :

$$
Z\left(a_{1}^{r}\right) \stackrel{\partial}{\longrightarrow} Z\left(a_{0}^{r}\right), \quad \text { where } \partial a_{1}^{r}=p^{u r} a_{0}^{r}
$$

( $p$ prime, $0<u_{1} \leqq u_{2} \leqq \cdots \leqq u_{n}$ ).

It can be verified that for each $r(r=1, \cdots, n)$ we have

$$
\begin{aligned}
H_{1}\left(K^{r}\right) & =0 ; \quad H_{0}\left(K^{r}\right)=Z_{p^{u_{r}}\left(\eta\left(a_{0}^{r}\right)\right) ;} \\
H_{1}\left(K^{r}, m\right) & =Z_{\left(m, p^{r_{r}}\right)}\left(\eta\left(s a_{1}^{r} \otimes 1_{m}\right)\right), \quad \text { where } s=\frac{m}{\left(m, p^{u_{r}}\right)} ; \\
H_{0}\left(K^{r}, m\right) & =Z_{\left(m, p^{u_{r}}\right)}\left(\eta\left(a_{0}^{r} \otimes 1_{m}\right)\right) .
\end{aligned}
$$

In dimension $1, \mu_{m k}^{m}$ and $\lambda_{m}^{m k}$ are the maps:

$$
\begin{aligned}
\text { ı: } Z_{\left(m, p^{\left.u_{r}\right)}\right.} \longrightarrow Z_{\left(m k, p^{\left.u_{r}\right)}\right.} & (k>0) ; \\
k \pi: Z_{\left(m k, p^{\left.u_{r}\right)}\right.} \longrightarrow Z_{\left(m, p^{u_{r}}\right)} & (k \geqq 0),
\end{aligned}
$$

where $k=k /\left(k, p^{u r} /\left(m, p^{u r}\right)\right) ; \imath$ and $\pi$ are the canonical injection and projection of cyclic groups. $\mu_{0}^{m}$ (which has degree -1 ) is the injenction

$$
\text { ı: } Z_{\left(m, p^{\left.u_{r}\right)}\right.} \longrightarrow Z_{p^{u_{r}}}
$$

In degree $0, \mu_{m k}^{m}$ and $\lambda_{m}^{m k}$ are the maps:

$$
\begin{aligned}
& k 1: Z_{\left(m, p^{u_{r}}\right)} \longrightarrow Z_{\left(m k, p^{u_{r}}\right)} \quad \quad(k>0 ; \bar{k} \text { as above); } \\
& \pi: Z_{\left(m k, p^{\left.u_{r}\right)}\right.} \longrightarrow Z_{\left(m, p^{\left.u_{r}\right)}\right.} .
\end{aligned}
$$

We shall use the following notation. Let $n \geqq 2$ be a fixed integer; for each integer $i, 0 \leqq i \leqq n$ let $J(i)$ be the set of all $n$-tuples $\left(j_{1}, \cdots, j_{n}\right)$ such that each $j_{k}=0$ or 1 and $\sum_{k=1}^{n} j_{k}=i$. Let $\bar{J}(i) \subset J(i)$ be the set of $n$-tuples with first entry 0 ; let $\tilde{J}(i) \subset J(i)$ be the set of $n$-tuples with first entry 1. For a given element $\left(j_{1}, \cdots, j_{n}\right)$ of $J(i)$, let $\varepsilon(k)=\sum_{r<k} j_{r}$ (for $\left.k=1, \cdots, n\right)$; denote by $\left(j_{1}, \cdots, 0_{k}, \cdots, j_{n}\right)$ [resp. $\left.\left(j_{1}, \cdots, 1_{k}, \cdots, j_{n}\right)\right]$ the $n$-tuple obtained by replacing $j_{k}$ with 0 [resp. 1] (for $k=1, \cdots, n$ ). 
If $j=\left(j_{1}, \cdots, j_{n}\right) \in J(i)$, let $x(j)=x\left(j_{1}, \cdots, j_{n}\right)$ be the generator $a_{j_{1}}^{1} \otimes a_{j_{2}}^{2} \otimes \cdots \otimes a_{J_{n}}^{n}$ of $\left(\otimes_{r=1}^{n} K^{r}\right)_{i}$. Then the boundary map

$$
\partial:\left(\bigotimes_{r=1}^{n} K^{r}\right)_{i} \longrightarrow\left(\bigotimes_{r=1}^{n} K^{r}\right)_{i-1}
$$

is given by

$$
\partial\left[x\left(j_{1}, \cdots, j_{n}\right)\right]=\sum_{k=1}^{n}(-1)^{\varepsilon(k)} j_{k} p^{u_{k}} x\left(j_{1}, \cdots, 0_{k}, \cdots, j_{n}\right) .
$$

[N. B. This statement is not quite accurate, since if $j_{k}=0$, then $x\left(j_{1}, \cdots, 0_{k}, \cdots, j_{n}\right)$ is a generator of $\left(\bigotimes_{r=1}^{n} K^{r}\right)_{i}$ and not of $\left(\bigotimes_{r=1}^{n} K^{r}\right)_{i-1}$; however, in this case the coefficient of $x\left(j_{1}, \cdots, 0_{k}, \cdots, j_{n}\right)$ in (1) is zero and we can consider $(-1)^{\varepsilon(k)} j_{k} p^{u_{k}} x\left(j_{1}, \cdots, 0_{k}, \cdots, j_{n}\right)=0$ as an element of $\left.\left(\bigotimes_{r=1}^{n} K^{r}\right)_{i-1} \cdot\right]$

It is clear that $\left(\bigotimes_{r=1}^{n} K^{r}\right)_{i}=\sum_{j \in J(i)} Z(x(j))$. Hence every element of $\left(\bigotimes_{r=1}^{n} K^{r}\right)_{i}$ can be written as a finite sum $\sum_{j \in J(i)} t(j) x(j)(t(j) \in Z)$. It follows from (1) that

$$
\partial\left[\sum_{j \in J(i)} t(j) x(j)\right]=\sum_{J(i-1)} y(j)
$$

where for $j=\left(j_{1}, \cdots, j_{n}\right) \in J(i-1)$,

$$
\begin{aligned}
y(j) & =y\left(j_{1}, \cdots, j_{n}\right) \\
& =\sum_{k=1}^{n}(-1)^{\varepsilon(k)}\left(1-j_{k}\right) p^{u_{k}} t\left(j_{1}, \cdots, 1_{k}, \cdots, j_{n}\right) x\left(j_{1}, \cdots, 1_{k}, \cdots, j_{n}\right) .
\end{aligned}
$$

There is the same sort of inaccuracy here as in (1) above and we use the same convention to eliminate it.]

If $i \leqq n-1$ and $j=\left(0, j_{2}, \cdots, j_{n}\right) \in \bar{J}(i)$ we define the element

$$
\bar{x}(j)=\bar{x}\left(0, j_{2}, \cdots, \bar{j}_{n}\right)=\sum_{j \in J(i)} t(j) x(j)
$$

of $\left(\bigotimes_{r=1}^{n} K^{r}\right)_{i}$ as follows:

$$
\begin{aligned}
& t(j)=1 ; \\
& t(j)=(-1)^{(\bar{\varepsilon}(k)+1)} p^{u_{k}-u_{1}} \quad(\vec{\varepsilon}(k) \text { determined by } j) \\
& \text { provided } j=\left(1, j_{2}, \cdots, j_{n}\right) \in J(i) \text { and } \\
& \quad \text { for some value } k, 1<k \leqq n, \\
& \quad j_{r}=j_{r} \text { for } 1<r \neq k \text { and } j_{k}=1, j_{k}=0 ; \\
& t(j)=0 \text { for all other } j \in J(i) .
\end{aligned}
$$

A direct calculation, using (2), shows that for any $j \in \bar{J}(i), \partial \bar{x}(j)=0$; hence $\eta(\bar{x}(\bar{j})) \in H_{i}\left(\bigotimes_{r=1}^{n} K^{r}\right)$. 
LEMMA 3.2. If $K^{1}, \cdots, K^{n}$ are elementary complexes as described above, then for each $i, 0 \leqq i \leqq n-1$,

$$
H_{i}\left(\bigotimes_{r=1}^{n} K^{r}\right)=\sum_{\bar{j} \in \bar{J}_{(i)}} Z_{p^{u_{1}}}(\eta(\bar{x}(\bar{j}))) .
$$

Proof. If $\sum_{j \in J(i)} t(j) x(j)$ is a cycle of $\left(\otimes_{r=1}^{n} K^{r}\right)_{i}$, then by (2) above we have for each $\left(j_{1}, \cdots, j_{n}\right) \in J(i-1)$ a relation:

$$
\sum_{k=1}^{n}(-1)^{\varepsilon(k)}\left(1-j_{k}\right) p^{u_{k}} t\left(j_{1}, \cdots, 1_{k}, \cdots, j_{n}\right)=0 .
$$

If $\left(1, j_{2}, \cdots, j_{n}\right) \in J(i)$, the relation (3) associated with $\left(0, j_{2}, \cdots, j_{i n}\right) \in J(i-1)$ is:

$$
p^{u_{1}} t\left(1, j_{2}, \cdots, j_{n}\right)+\sum_{k=2}^{n}(-1)^{\varepsilon(k)}\left(1-j_{k}\right) p^{u_{k}} t\left(0, j_{2}, \cdots, 1_{k}, \cdots, j_{n}\right)=0 ;
$$

hence we have:

$$
t\left(1, j_{2}, \cdots, j_{n}\right)=-\sum_{k=2}^{n}(-1)^{\varepsilon(k)}\left(1-j_{k}\right) p^{u_{k}-u_{1}} t\left(0, j_{2}, \cdots, 1_{k}, \cdots, j_{n}\right) .
$$

Relation (4) shows that for every cycle $\sum_{j \in J(t)} t(j) x(j)$ of $\left(\bigotimes_{r=1}^{n} K^{r}\right)_{i}$ the coefficients $t(j)$ are completely determined by the integers $t(j)$ with $j \in \bar{J}(i)$. Thus in order to obtain a set of generators for the cycles of $\left(\otimes_{r=1}^{n} K^{r}\right)_{i}$, we proceed as follows. For each $j \in \bar{J}(i)$, set $t(j)=1$ and $t(j)=0$ for $j \in \bar{J}(i), j \neq j$; then use relation (4) above to determine the values of $t(j)$ for $j \in \tilde{J}(i)$ in order that $\sum_{j \in J(i)} t(j) x(j)$ be a cycle. But a careful examination shows that this is precisely how the elements $\bar{x}(j)[j \in \bar{J}(i)]$ were defined above. In other words, $\{\bar{x}(j) \mid j \in \bar{J}(i)\}$ is a set of generators for the cycles of $\left(\bigotimes_{r=1}^{n} K^{r}\right)_{i}$.

The boundaries in $\left(\bigotimes_{r=1}^{n} K^{r}\right)_{i}$ are generated by the images of the elements $x(j)[j \in J(i+1)]$. Since in each complex $K^{r}(r=1, \cdots, n)$ the boundary operator involves multiplication by $p^{u_{r}}=p^{u_{1}} \cdot p^{u_{r}-u_{1}}$ it follows that a necessary condition for an element $\sum_{j \in J(i)} t(j) x(j)$ to be a boundary is that $p^{u_{1}} \mid t(j)$ for every $j \in J(i)$. Consequently, all of the cycles $\bar{x}(j)[j \in \bar{J}(i)]$ have distinct homology classes since the difference of any two would have some coefficients $t(j)= \pm 1$ (and $p^{u_{1}}>1$ since $\left.u_{1}>0\right)$. Furthermore, if $j=\left(0, j_{2}, \cdots, j_{n}\right) \in \bar{J}(i)$, then $\left(1, j_{2}, \cdots, j_{n}\right) \in J(i+1)$ and $\partial x\left(1, j_{2}, \cdots, j_{n}\right)=p^{u_{1}} \bar{x}(j)$. Since no smaller multiple of $\bar{x}(\bar{j})$ can be a boundary the conclusion of the lemma follows.

Now for each $i, 1 \leqq i \leqq n$, let $j=\left(1, j_{2}, \cdots, j_{n}\right) \in \tilde{J}(i)$ and define $B_{j}{ }^{i}$ to be the group

$$
H_{1}\left(K^{1}, p^{u_{1}}\right) \otimes H_{j_{2}}\left(K^{2}, p^{u_{1}}\right) \otimes \cdots \otimes H_{j_{n}}\left(K^{n}, p^{u_{1}}\right)=\bigotimes_{k=1}^{n} H_{j_{k}}\left(K^{k}, p^{u_{1}}\right) .
$$

Let $B(i)=\sum_{j \in \widetilde{J}(i)} B_{j}^{i}$; let $B(0)=\bigotimes_{r=1}^{n} H_{0}\left(K^{\eta}\right)$; and let $B(i)=0$ for all other values of $i$. Note that $B(0)$ is a cyclic group of order $p^{u_{1}}$ generated by 


$$
\eta\left(a_{0}^{1}\right) \otimes \eta\left(a_{0}^{2}\right) \otimes \cdots \otimes \eta\left(a_{0}^{n}\right)
$$

and for $1 \leqq i \leqq n, j=\left(j_{1}, \cdots, j_{n}\right) \in \tilde{J}(i), B_{j}^{i}$ is a cyclic group of order $p^{u_{1}}$ generated by

$$
\eta\left(a_{j_{1}}^{1} \otimes 1_{p} u_{1}\right) \otimes \eta\left(a_{j_{2}}^{2} \otimes 1_{p} u_{1}\right) \otimes \cdots \otimes \eta\left(a_{i_{n}}^{n} \otimes 1_{p} u_{1}\right)
$$

we shall denote this generator by $y\left(j_{1}, \cdots, j_{n}\right)[j \in \tilde{J}(i)]$.

LEMMA 3.3. If $K^{1}, \cdots, K^{n}$ are elementary complexes as described above, then the group $\sum_{i \neq 1} B(i)$ is a complete set of representatives of the quotient group $\sum_{m \geqq 0}\left[\bigotimes_{r=1}^{n} H\left(K^{r}, m\right)\right] / S$ (where $S$ is the subgroup of Definition 2.1). In other words, every element of the group $\sum_{m \geqq 0}\left[\bigotimes_{r=1}^{n} H\left(K^{r}, m\right)\right]$ can be identified (modulo the subgroup $S$ ) with an element of the group $\sum_{i \neq 1} B(i)$.

Proof. By using the relations (1) of Definition 2.1 it is immediately verified that for $m>0$ every element of $\bigotimes_{r=1}^{n} H_{0}\left(K^{r}, m\right)$ is congruent to zero (modulo $S$ ) and every element of the groups

$$
H_{0}\left(K^{1}, m\right) \otimes \cdots \otimes H_{0}\left(K^{k-1}, m\right) \otimes H_{1}\left(K^{k}, m\right) \otimes H_{0}\left(K^{k+1}, m\right) \otimes \cdots \otimes H_{0}\left(K^{n}, m\right)
$$

(for $k=1,2, \cdots, n)$ is identified $(\bmod S)$ with an element of $B(0)$.

For $i \neq 0,1$ we proceed as in the proof of Theorem 2.2 in the Appendix of [3], using greatest common divisors. It follows that every generator of

$$
\sum_{m \geqq 0}\left[\bigotimes_{r=1}^{n} H\left(K^{r}, m\right)\right]
$$

of total degree $i$ is identified $(\bmod S)$ with an element of the group

$$
\sum_{j \in J(i)} H_{j_{1}}\left(K^{1}, C(j)\right) \otimes \cdots \otimes H_{j_{n}}\left(K^{n}, C(j)\right)
$$

where $j=\left(j_{1}, \cdots, j_{k}\right) \in J(i)$ and $C(j)$ is the greatest common divisor of $\left\{p^{u_{k}} \mid j_{k}=1\right\}$.

We now use relation (2) of Definition 2.1 to show that every element of the group (5) can be identified (mod $S$ ) with an element of the group $B(i)$. Recall that $J(i)=\tilde{J}(i) \cup \tilde{J}(i)$. If $j=\left(j_{1}, \cdots, j_{n}\right) \in \tilde{J}(i)$, then $j_{1}=1$ by definition and hence $C(j)=p^{u_{1}}$; therefore

$$
H_{j_{1}}\left(K^{1}, C(j)\right) \otimes \cdots \otimes H_{j_{n}}\left(K^{n}, C(j)\right)
$$

is just the group $B_{j}^{i} \subset B(i)$.

If $j=\left(j_{1}, \cdots, j_{n}\right) \in \bar{J}_{i}$, then $j_{1}=0$. Consider the map

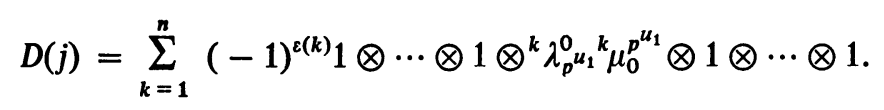

Apply $D(j)$ to the generator $y\left(1, j_{2}, \cdots, j_{n}\right) \in B_{\left(1, j_{2}, \ldots, j .\right)}^{i+1}$. Note that for $\eta\left(a_{1}^{k} \otimes 1_{p}^{u_{1}}\right) \in H_{1}\left(K^{k}, p^{u_{1}}\right)$, 


$$
{ }^{k} \lambda_{p^{u_{1}}}^{u^{k}} \mu_{0}^{p_{1}{ }_{1}} \eta\left(a_{1}^{k} \otimes 1_{p^{u_{1}}}\right)=\eta\left(a_{0}^{k} \otimes 1_{p^{u_{1}}}\right) \in H_{0}\left(K^{k}, p^{u_{1}}\right) ;
$$

also

$$
{ }^{k} \lambda_{p^{u_{1}}}{ }^{k} \mu_{0}^{p^{u}{ }^{1}} \eta\left(a_{0}^{k} \otimes 1{ }_{p}^{u_{1}}\right)=0
$$

It follows that

$$
D(j)\left[y\left(1, j_{2}, \cdots, j_{n}\right)\right]=y\left(0, j_{2}, \cdots, j_{n}\right)+\sum_{k=2}^{n}(-1)^{\varepsilon(k)} j_{k} y\left(1, j_{2}, \cdots, 0_{k}, \cdots, j\right) .
$$

But by relation (2) of Definition 2.1

$$
D(j)\left[y\left(1, j_{2}, \cdots, j_{n}\right)\right] \equiv 0(\bmod S) .
$$

Consequently each $y(j)[j \in \bar{J}(i)]$ is identified $(\bmod S)$ with a linear combination of elements $y(\tilde{j})[\tilde{j} \in \tilde{J}(i)]$. Since each $y(\tilde{J}) \in B(i)$, the lemma is proved.

Lemma 3.3 shows that the group

$$
\bigotimes_{r=1}^{n}\left\{H\left(K^{r}, m\right)\right\}=\sum_{m \geq 0}\left[\bigotimes_{r=1}^{n} H\left(K^{r}, m\right)\right] / S
$$

is (a quotient group of) the group $\sum_{i \neq 1} B(i)$, where $B(i)(i>0)$ is generated by the elements $y(j)[j \in \tilde{J}(i)]$.

It is clear that the map $F$ induces an isomorphism

$$
B(0)=\bigotimes_{r=1}^{n} H\left(K^{r}\right) \stackrel{\alpha}{\longrightarrow} H_{0}\left(\bigotimes_{r=1}^{n} K^{r}\right) .
$$

For $i>1$ a direct calculation shows that for each $j=\left(1, j_{2}, \cdots, j_{n}\right) \in \tilde{J}(i)$,

$$
F[y(j)]=\eta\left(\bar{x}\left(0, j_{2}, \cdots, j_{n}\right)\right) ;
$$

the element $\eta\left(\bar{x}\left(0, j_{2}, \cdots, j_{i}\right)\right)$, as was shown above, is a generator of $H_{t-1}\left(\bigotimes_{r=1}^{n} K^{\prime}\right)$.

Since for any $\left(0, j_{2}, \cdots, j_{n}\right) \in \bar{J}(i-1)$, we have $\left(1, j_{2}, \cdots, j_{n}\right) \in \tilde{J}(i)$ and

$$
F\left[y\left(1, j_{2}, \cdots, j_{n}\right)\right]=\eta\left(\bar{x}\left(0, j_{2}, \cdots, j_{n}\right)\right)
$$

it follows that $F$ induces an isomorphism of $B(i)$ onto $H_{i-1}\left(\bigotimes_{r=1}^{n} K^{r}\right)$. Consequently $F$ induces an isomorphism

$$
\sum_{i \neq 1} B(i) \cong H\left(\bigotimes_{r=1}^{n} K^{r}\right) .
$$

It follows that the group $\bigotimes_{r=1}^{n}\left\{H\left(K^{r}, m\right)\right\}$ must be precisely the group $\sum_{i \neq 1} B(i)$ and that the map $F$ induced by $F$ on the quotient

$$
\sum_{m \geqq 0}\left[\bigotimes_{r=1}^{n} H\left(K^{\prime}, m\right)\right] / S=\bigotimes_{r=1}^{n}\left\{H\left(K^{r}, m\right)\right\}
$$


is an isomorphism of graded groups:

$$
F: \bigotimes_{r=1}^{n}\left\{H\left(K^{r}, m\right)\right\} \cong H\left(\bigotimes_{r=1}^{n} K^{r}\right) \text {. }
$$

This completes the proof of Theorem 3.1.

We remark that all of the constructions for products given for $n=3$ in the last part of [3] now carry over without difficulty to the case of arbitrary $n$.

4. The Universal Coefficient Theorem. The next step in the proof of Theorem 1.1 is to replace each of the homology spectra $\left\{H\left(K^{r}, m\right)\right\}(r=1, \cdots, n)$ with a suitable isomorphic Bockstein spectrum. We do so as follows.

TheOrem 4.1 (UnIVersal Coefficient Theorem). If $K$ is a chain complex of torsion-free abelian groups and $A$ is an abelian group, then there is a split exact sequence:

$$
0 \longrightarrow H(K) \otimes A \underset{\bar{\alpha}}{\stackrel{\alpha}{\rightleftarrows}} H(K \otimes A) \underset{\bar{\beta}}{\stackrel{\beta}{\rightleftarrows}} \operatorname{Tor}(H(K), A) \longrightarrow 0,
$$

where $\alpha[\eta(u) \otimes a]=\eta(u \otimes a)$ for $u \in Z(K), a \in A$. The maps $\alpha$ and $\beta$ are natural in $K$ and $A$; the maps $\bar{\alpha}$ and $\bar{\beta}$ are natural in $A$ (but not in $K$ ).

Except for the statement about the naturality of $\bar{\alpha}$ and $\bar{\beta}$ (the proof of which can easily be supplied) this is just Theorem V.11 of MacLane [5]. If $A$ is any abelian group and $m>0$ is an integer, ${ }_{m} A$ is the subgroup $\{a \in A \mid m a=0\}$; we set ${ }_{0} A=0$.

COROLLARY 4.2. If $K$ is a chain complex of torsion-free abelian groups, then for each $m>0$ there is a split exact sequence:

$$
0 \longrightarrow H(K) / m H(K) \stackrel{\pi_{*}}{\longrightarrow} H(K, m) \stackrel{\delta_{0}^{m}}{\longrightarrow}{ }_{m} H(K) \longrightarrow 0,
$$

where $\delta_{0}^{m}$ is the Bockstein connecting homomorphism and $\pi_{*}[\eta(u)+m H(K)]$ $=\eta\left(u \otimes 1_{m}\right)[u \in Z(K)]$.

Proof. In Theorem 4.1 let $A=Z_{m}$; then $H(K) \otimes A$ is the group

$$
H(K) \otimes Z_{m} \cong H(K) / m H(K) \text {. }
$$

It follows that the first map in the displayed sequence is $\pi_{*}$ as stated. Similarly, $\operatorname{Tor}(H(K), A)=\operatorname{Tor}\left(H(K), Z_{m}\right) \cong{ }_{m} H(K)$. The fact that the second map in the sequence is $\delta_{0}^{m}$ follows from the description of the map $\beta$ (of Theorem 4.1) given in Proposition V.10.6 of MacLane [5].

Hereafter we shall denote the group $H(K) / m H(K)$ by $H(K)_{m}$. 
COROLlaRY 4.3. If $K$ is a chain complex of torsion-free abelian groups, then for each $m \geqq 0$ there is an isomorphism

$$
\zeta_{m}: H(K, m) \cong H(K)_{m} \oplus_{m} H(K) \text {. }
$$

For $m>0$ this is merely a restatement of Corollary 4.2 in a more convenient form. $\zeta_{0}$ is the identity map on $H(K)_{0} \oplus{ }_{0} H(K)=H(K) \oplus 0=H(K)$. Note that for $m>0,\left.\zeta^{-1}\right|_{m} H(K)$ is the right inverse of $\delta_{0}^{m}$ (i.e., the map which splits the sequence in Corollary 3.2) and therefore $\zeta^{-1}:{ }_{m} H_{p-1}(K) \rightarrow H_{p}(K, m)$.

The collection $\left\{H(K)_{m} \mid m \geqq 0\right\}=\left\{H(K)_{m}\right\}$ can be considered as a Bockstein spectrum with maps $\bar{\lambda}_{m}^{m k}$ and $\bar{\mu}_{m k}^{m}$ induced by the identity map on $H(K)$ and by multiplication by $k$ in $H(K)$, respectively. Similarly $\left\{{ }_{m} H(K)\right\}$ is the Bockstein spectrum with maps $\hat{\lambda}_{m}^{m k}$ and $\hat{\mu}_{m k}^{m}$ induced by multiplication by $k$ in $H(K)$ and by the identity map on $H(K)$, respectively. We would like to define maps $\lambda$ and $\mu$ on $H(K)_{m} \oplus_{m} H(K)$ in such a way that it becomes a Bockstein spectrum.

DEFINITION 4.4. If $K$ is a chain complex of torsion-free abelian groups, (for convenience) let $U_{m}=H(K)_{m} \oplus{ }_{m} H(K)\left[m \geqq 0 ; U_{0}=H(K)\right]$. Let $\lambda_{m}^{m k}: U_{m k} \rightarrow U_{m}$ and $\mu_{m k}^{m}: U_{m} \rightarrow U_{m k}(m k>0)$ be the maps:

and

$$
\bar{\lambda}_{m}^{m k} \oplus \hat{\lambda}_{m}^{m k}: H(K)_{m k} \oplus_{m k} H(K) \longrightarrow H(K)_{m} \oplus_{m} H(K)
$$

$$
\bar{\mu}_{m k}^{m} \oplus \hat{\mu}_{m k}^{m}: H(K)_{m} \oplus_{m} H(K) \longrightarrow H(K)_{m k} \oplus_{m k} H(K) .
$$

Let $\lambda_{m}^{0}: U_{0} \rightarrow U_{m}(m>0)$ be the composition:

$$
H(K) \stackrel{\lambda_{m}^{0}}{\longrightarrow} H(K)_{m} \stackrel{\subset}{\longrightarrow} H(K)_{m} \oplus_{m} H(K) .
$$

Let $\mu_{0}^{m}: U_{m} \rightarrow U_{0}(m>0)$ be the map:

$$
\left(\bar{\mu}_{0}^{m}+\imath_{0}^{m}\right): H(K)_{m} \oplus_{m} H(K) \longrightarrow H(K),
$$

where $r_{0}^{m}:{ }_{m} H(K) \rightarrow H(K)$ is the inclusion map; note that $\bar{\mu}_{0}^{m}$ is the zero map. Finally let $\lambda_{0}^{0}=\mu_{0}^{0}$ identity on $U_{0}=H(K)$.

Proposition 4.5. If $K$ is a chain complex of torsion-free abelian groups, then $\left\{H(K)_{m} \oplus_{m} H(K)\right\}$ with maps $\lambda$ and $\mu$ as in Definition 4.4 is a Bockstein spectrum. Furthermore, if $\zeta_{m}(m \geqq 0)$ is as in Corollary 4.3 , then

$$
\zeta=\left\{\zeta_{m}\right\}:\{H(K, m)\} \longrightarrow\left\{H(K)_{m} \oplus_{m} H(K)\right\}
$$

is an isomorphism of Bockstein spectra.

The proof of the first part is straightforward and is omitted. The last statement means that $\zeta$ commutes with the various $\lambda$ 's and $\mu$ 's; it follows from the commutativity of the appropriate diagrams (and this depends on the naturality statement in Theorem 4.1). Note that the spectrum $\left\{H(K)_{m} \oplus_{m} H(K)\right\}$ is not the direct sum spectrum $\left\{H(K)_{m}\right\} \oplus\left\{_{m} H(K)\right\}$. For the map $\mu_{0}^{m}$ in this direct sum is given by 
$\left(\bar{\mu}_{0}^{m}+\hat{\mu}_{0}^{m}\right)=(0+0)=0$; but the map $\mu_{0}^{m}=\left(\bar{\mu}_{0}^{m}+\imath_{0}^{m}\right)$ in $\left\{H(K)_{m} \oplus{ }_{m} H(K)\right\}$ need not be zero.

5. The Multiple Künneth Theorem. We wish to obtain a conceptual description of the derived functors $\operatorname{Mult}_{i}\left(A^{1}, \cdots, A^{n}\right)$. First, however, we need some notation.

The symbols ${ }_{m} A$ and $A_{n}$ have been used above. We now wish to "combine", these symbols in the following manner. If $A$ is an abelian group and $(m, n)$ is a pair of non-negative integers, one of which is zero and the other nonzero, we denote by ${ }_{m} A_{n}$ either the group ${ }_{m} A$ (if $m \neq 0$ ) or the group $A_{n}=A / n A($ if $n \neq 0$ ) as the case may be.

Suppose $0 \leqq i \leqq n$ and let $J(i)$ be as above. If $j=\left(j_{1}, \cdots, j_{n}\right) \in J(i), A^{1}, \cdots, A^{n}$ are abelian groups and $m>0$ is an integer, then we denote by $A_{(j)}^{1} \otimes A_{(j)}^{2} \otimes \cdots \otimes A_{(j)}^{n}=\bigotimes_{r=1}^{n} A_{(j)}^{r}$ the group

$$
j_{1 m} A^{1}{ }_{\left(1-j_{1}\right) m} \otimes j_{j_{2} m} A_{\left(1-j_{2}\right) m}^{2} \otimes \cdots \otimes_{j_{n} m} A_{\left(1-j_{n}\right) m}^{n}
$$

If $m=0$, we adopt the convention that ${ }_{j_{k} m} A_{\left(1-j_{k}\right) m}^{k}=0$ if $j_{k}=1$, and $=A^{k}$ if $j_{k}=0$. Then we can denote by $\left\{A_{(j)}^{k}\right\}$ the Bockstein spectrum $\left\{{ }_{j_{k} m} A_{\left(1-j_{k}\right) m}^{k}\right\}$, which will be either the spectrum $\left\{{ }_{m} A^{k}\right\}\left(j_{k}=1\right)$ or the spectrum $\left\{A_{m}^{k}\right\}\left(j_{k}=0\right)$. We denote by $\left\{A_{(j)}^{1}\right\} \otimes\left\{A_{(j)}^{2}\right\} \otimes \cdots \otimes\left\{A_{(j)}^{n}\right\}=\bigotimes_{r=1}^{n}\left\{A_{(j)}^{r}\right\}$ the tensor product of these Bockstein spectra. Thus

$$
\bigotimes_{r=1}^{n}\left\{A_{(j)}^{r}\right\}=\left[\sum_{m \geqq 0} A_{(j)}^{1} \otimes \cdots \otimes A_{(j)}^{n}\right] / S
$$

( $S$ as in Definition 2.1).

THEOREM 5.1. If $A^{1}, \cdots, A^{n}$ are abelian groups then $\operatorname{Mult}_{0}\left(A^{1}, \cdots, A^{n}\right)$ $=\bigotimes_{r=1}^{::} A^{r}$ and for $0<k \leqq n-1$

$$
\operatorname{Mult}_{k}\left(A^{1}, \cdots, A^{n}\right)=\left[\sum_{j \in J(k+1)}\left\{A_{(j)}^{1}\right\} \otimes \cdots \otimes\left\{A_{(j)}^{n}\right\}\right] / \tilde{E}(k),
$$

where $\tilde{E}(k)=0$ for $k=n-1$ and otherwise $\tilde{E}(k)$ is the image of the group $\Sigma_{j \in J(k+2)}\left\{A_{(j)}^{1}\right\} \otimes \cdots \otimes\left\{A_{(j)}^{n}\right\}$ under the map (induced on a summand by)

$$
\sum_{m \geqq 0} \sum_{r=1}^{n} 1 \otimes \cdots \otimes 1 \otimes j_{r} g_{r} \otimes 1 \otimes \cdots \otimes 1,
$$

where for each $m \geqq 0, j_{r} g_{r}=0$ if $j_{r}=0$ and if $j_{r}=1, j_{r} g_{r}$ is the composition:

$$
{ }_{m} A^{r} \stackrel{\subset}{\longrightarrow} A^{r} \longrightarrow A^{r} / m A^{r}=A_{m}^{r} \text {. }
$$

Proof. For each $r, 1 \leqq r \leqq n$, let $K^{r}$ be a free resolution of $A^{r}$. By definition $\operatorname{Mult}_{i}\left(A^{1}, \cdots, A^{n}\right)$ is the group $H_{i}\left(\bigotimes_{r=1}^{n} K^{r}\right)$.

By Theorem 3.1 and Proposition 4.5 there are isomorphisms $F$ and $\zeta=\zeta \otimes \cdots \otimes \zeta$ such that 


$$
\zeta F^{-1}: H\left(\bigotimes_{r=1}^{n} K^{r}\right) \cong \bigotimes_{r=1}^{n}\left\{H\left(K^{r}, m\right)\right\} \cong \bigotimes_{r=1}^{n}\left\{H\left(K^{r}\right)_{m} \oplus_{m} H\left(K^{r}\right)\right\}
$$

The latter group is the quotient group of the group

$$
\sum_{i=0}^{n} \sum_{J \in J(i)} \sum_{m \geqq 0} H\left(K^{1}\right)_{(j)} \otimes \cdots \otimes H\left(K^{n}\right)_{(j)}
$$

by the subgroup $S$ (as in Definition 2.1).

It follows immediately from the definition of the maps $\lambda$ and $\mu$ in $\left\{H\left(K^{r}\right)_{m} \oplus_{m} H\left(K^{r}\right)\right\}(r=1, \cdots, n)$ and from the relations (1) of Definition 2.1 that every element of the subgroup

$$
\sum_{i=0}^{1} \sum_{j \in J(i)} \sum_{m \geqq 0} H\left(K^{1}\right)_{(j)} \otimes \cdots \otimes H\left(K^{n}\right)_{(j)}
$$

is identified (modulo $S$ ) with an element of the group $\bigotimes_{r=1}^{n} H\left(K^{r}\right)$. Consequently, $H\left(\otimes_{r=1}^{n} K^{r}\right)$ is isomorphic to the quotient group of the group

$$
\bigotimes_{r=1}^{\imath} H\left(K^{r}\right) \oplus \sum_{i=2}^{n} \sum_{j \in J(i)} \sum_{m>0} H\left(K^{1}\right)_{(j)} \otimes \cdots \otimes H\left(K^{n}\right)_{(j)}
$$

by the subgroup (induced by) $S$.

Since the map $F$ is induced by the map $F$ which has degree -1 (for $m>0$ ) and since

$$
\zeta^{-1}:{ }_{m} H_{p-1}\left(K^{\eta}\right) \longrightarrow H_{p}\left(K^{r}, m\right) \quad(r=1,2, \cdots, n),
$$

it follows that $H_{k}\left(\bigotimes_{r=1}^{n} K^{r}\right)(k=0,1, \cdots, n-1)$ is isomorphic to the quotient group of the group

$$
\sum H_{p_{1}}\left(K^{1}\right) \otimes \cdots \otimes H_{p_{n}}\left(K^{n}\right)
$$

$$
\oplus \sum_{i=2}^{n} \sum_{j \in J(i)} \sum \sum_{m \geqq 0} H_{p_{2}}\left(K^{1}\right)_{(j)} \otimes \cdots \otimes H_{p_{n}}\left(K^{n}\right)_{(j)}
$$

(where the first sum is over all $n$-tuples $\left(p_{1}, \cdots, p_{n}\right)$ such that $\sum_{r=1} p_{r}=k$ and the fourth sum is over all $n$-tuples such that $\left.\sum_{r=1}^{n} p_{r}=k-i+1\right)$ modulo the relations induced by the subgroup $S$.

The relations induced by $S$ are of two forms (cf. Definition 2.1). Those of the form (1) are precisely those needed to reduce the group (1) above to the group:

$$
\sum_{M}\left(\bigotimes_{r=1}^{n} H_{p_{r}}\left(K^{r}\right)\right) \oplus \sum_{i=2}^{n} \sum_{j \in J(i)} \sum\left\{H_{p_{1}}\left(K^{1}\right)_{(j)}\right\} \otimes \cdots \otimes\left\{H_{p_{n}}\left(K^{i}\right)_{(j)}\right\} \text {, }
$$

(first and fourth sums as in (1)).

The subgroup $E$ induced by the relations of the form (2) (in Definition 2.1) is the image of the group 


$$
\sum_{i=2}^{n-1} \sum_{j \in J(i+1)} \sum \sum_{m>0} H_{p_{2}}\left(K^{1}\right)_{(j)} \otimes \cdots \otimes H_{p_{n}}\left(K^{\prime \prime}\right)_{(j)}
$$

(the third sum being taken over all $n$-tuples such that $\sum_{r=1}^{n} p_{r}=k-i+1$ ) under the maps

$$
\sum_{r=1}^{n}(-1)^{*} 1 \otimes \cdots \otimes 1 \otimes{ }^{r} \lambda_{m}^{0}{ }^{r} \mu_{m}^{0} \otimes 1 \otimes \cdots \otimes 1 .
$$

Thus we conclude that $\operatorname{Mult}_{\dot{k}}\left(A^{1}, \cdots, A^{n}\right)=H_{k}\left(\bigotimes_{r=1}^{n} K^{r}\right)$ is isomorphic to the group

(2) $\Sigma\left(\bigotimes_{r=1}^{n} H_{p_{r}}\left(K^{r}\right)\right) \otimes\left[\sum_{i=2} \sum_{j \in J(i)} \sum\left\{H_{p_{1}}\left(K^{1}\right)_{(j)}\right\} \otimes \cdots \otimes\left\{H_{p_{n}}\left(K^{n}\right)_{(j)}\right\}\right] / E$

(first and fourth sums as in (1)).

However, $K^{r}$ is a free resolution of $A^{r}(r=1, \cdots, n)$; hence $H_{i}\left(K^{r}\right)=0$ for $i>0$ and $H_{0}\left(K^{r}\right) \cong A^{r}$. Applying these isomorphisms to the group (2), we obtain for $k=0$,

$$
\operatorname{Mult}_{0}\left(A^{1}, \cdots, A^{n}\right) \cong H_{0}\left(\bigotimes_{r=1}^{n} K^{r}\right)=\bigotimes_{r=1}^{n} A^{r}
$$

(in this case the second summand of (2) is empty). For $0<k \leqq n-1$, the only nonzero case is that in which $\left(p_{1}, \cdots, p_{n}\right)=(0, \cdots, 0)$; hence the only nontrivial summand in (2) is that in which $i=k+1$ and we obtain

$$
\operatorname{Mult}_{k}\left(A^{1}, \cdots, A^{n}\right) \cong\left[\sum_{j \in J(k+1)}\left\{A_{(j)}^{1}\right\} \otimes \cdots \otimes\left\{A_{(j)}^{n}\right\}\right] / E(k),
$$

where $E(k)$ is the image of $E$ under the isomorphism. Under the isomorphism the maps

$$
{ }^{r} \lambda_{m}^{0 r} \mu_{0}^{m}: H\left(K^{r}, m\right) \longrightarrow H\left(K^{r}\right) \longrightarrow H\left(K^{r}, m\right)
$$

become the maps $j_{r} g_{r}$ as in the statement of the theorem. It is easily verified that the group $E(k)$ obtained here is precisely the group $\widetilde{E}(k)$ in the statement of the theorem. [Note that $E(n-1)=0$ since, by definition, there are no elements in $J(n+1) \cdot]$

We are finally in a position for the

Proof of Theorem 1.1. We shall first assume that all $n$ complexes are torsionfree. In this case we may repeat the first part of the proof of Theorem 5.1 verbatim (since all of the calculations up to the statement of the group (2) depend only on the fact that the $K^{r}$ are torsion-free). Thus we have that $H_{k}\left(\bigotimes_{r=1}^{n} K^{r}\right)$ is isomorphic to the group

$$
\sum\left(\bigotimes_{r=1}^{n} H_{p_{r}}\left(K^{r}\right)\right) \oplus\left[\sum_{i=2}^{n} \sum_{j \in J(i)} \sum\left\{H_{p_{1}}\left(K^{1}\right)_{(j)}\right\} \otimes \cdots \otimes\left\{H_{p_{n}}\left(K^{n}\right)_{(j)}\right\}\right] / E
$$


(where the first sum is over all $n$-tuples such that $\sum_{r=1}^{n} p_{r}=k$ and the fourth over all $n$-tuples such that $\left.\sum_{r=1}^{n} p_{r}=k-i+1\right)$. For each $n$-tuple $\left(p_{1}, \cdots, p_{n}\right)$ we now apply Theorem 5.1 (and change the index $i$ to $i-1$ ) and $\left(2^{\prime}\right)$ becomes

$$
\sum \operatorname{Mult}_{0}\left(H_{p_{1}}\left(K^{1}\right), \cdots, H_{p_{n}}\left(K^{n}\right)\right) \oplus \sum_{i=1}^{n-1} \sum \operatorname{Mult}_{i}\left(H_{p_{1}}\left(K^{1}\right), \cdots, H_{p_{n}}\left(K^{n}\right)\right)
$$

(where the first sum is over all $n$-tuples such that $\sum_{r=1}^{n} p_{r}=k$ and the third over all such that $\left.\sum_{r=1}^{n} p_{r}=k-i\right)$. [Recall that the group $E$ of $\left(2^{\prime}\right)$ was originally defined on the homogeneous summands for each $n$-tuple $\left(p_{1}, \cdots, p_{n}\right)$; hence in this case $E=\Sigma \widetilde{E}(k)$.$] If we sum over k$, we have

$$
H\left(\bigotimes_{r=1}^{n} K^{r}\right) \cong \sum_{i=0}^{n-1} \operatorname{Mult}_{i}\left(H\left(K^{1}\right), \cdots, H\left(K^{n}\right)\right)
$$

This isomorphism induces maps

$$
f_{0}: \operatorname{Mult}_{0}\left(H\left(K^{1}\right), \cdots, H\left(K^{n}\right)\right) \longrightarrow H\left(\bigotimes_{r=1}^{n} K^{r}\right) ;
$$

$f_{i}: \operatorname{Mult}_{i}\left(H\left(K^{1}\right), \cdots, H\left(K^{n}\right)\right) \longrightarrow H\left(\bigotimes_{r=1}^{n} K^{r}\right) / \sum_{j<i} \operatorname{Mult}_{j}\left(H\left(K^{1}\right), \cdots, H\left(K^{n}\right)\right)$,

the latter being Cokernel $f_{i-1}$. The map $f_{0}$ is just the homology product

$$
\alpha: \bigotimes_{r=1}^{n} H\left(K^{r}\right) \longrightarrow H\left(\bigotimes_{r=1}^{n} K^{r}\right)
$$

and is known to be natural. An examination of the isomorphism (3) shows that the maps $f_{i}$ are induced by the composition $F \bar{\zeta}^{-1}$ [see p. 15]. The map $\bar{\zeta}^{-1}$ is the map $\zeta^{-1} \otimes \cdots \otimes \zeta^{-1}$, where for each $r(r=1, \cdots, n)$

$$
\zeta:\left\{H\left(K^{r}, m\right)\right\} \cong\left\{H\left(K^{r}\right)_{m} \oplus_{m} H\left(K^{r}\right)\right\}
$$

[see Proposition 4.5]. From Corollary 4.2 it follows that $\zeta^{-1}$ is natural on $H\left(K^{r}\right)_{m}$, but not on ${ }_{m} H\left(K^{\prime}\right)$. However, the isomorphism induced by

$$
\zeta^{-1}:{ }_{m} H\left(K^{r}\right) \cong H\left(K^{r}, m\right) / H\left(K^{r}\right)_{m}
$$

is natural [it is the inverse of a natural isomorphism-cf. the naturality statements in Theorem 4.1]. Thus $F^{\zeta^{-1}}$ is natural up to a certain indeterminacy involving the $H\left(K^{\prime}\right)_{m}$. It follows from this fact that the maps $f_{i}$ are natural for $i>0$.

This completes the proof of Theorem 1.1; for as remarked in $\S 1$ we need only apply the results to the case of the double complex projective resolutions $R^{1}, \cdots, R^{r}$ (of complexes $\left.K^{1}, \cdots, K^{r}\right)$. For each $r, H\left(R^{r}\right) \cong H\left(K^{r}\right)$ and $\mathscr{L}\left(K^{1} \otimes \cdots \otimes K^{n}\right)$ $=H\left(\bigotimes_{r=1}^{n} R^{r}\right)$.

Finally we assert that the functors $\operatorname{Mult}_{i}\left(A^{1}, \cdots, A^{n}\right)$ defined inductively in Bockstein [9] (and denoted hereafter by $B$-Mult to avoid confusion) are in fact 
the functors Mult $_{i-1}\left(A^{1}, \cdots, A^{n}\right)$ (as defined in $\S 1$ ). Bockstein first defines $\operatorname{Tor}\left(A^{1}, \cdots, A^{k}\right)$ to be the abelian group generated by all symbols $\tau_{h}\left(a^{1}, \cdots, a^{k}\right)$, where $a^{i} \in A^{i}$ and $h a^{i}=0(i=1, \cdots, k)$, subject to the relations:

(1) Additivity in each argument $a^{i}(i=1, \cdots, k)$;

$$
\tau_{h}\left(m a^{1}, \cdots, m a^{i-1}, a^{i}, m a^{i+1}, \cdots, m a^{k}\right)=\tau_{m h}\left(a^{1}, \cdots, a^{\dot{k}}\right) \quad(i=1, \cdots, k)
$$

(i.e. (1) and (2) are required to hold whenever both sides of the equation are defined). $B$-Mult $\left(A^{1}, \cdots, A^{n}\right)$ is then defined to be the abelian group

$$
\left[\Sigma \operatorname{Tor}\left(A^{s_{1}}, \cdots, A^{s_{i}}\right) \otimes A^{r_{1}} \otimes \cdots \otimes A^{r_{-1-i}}\right] / E(i),
$$

where the sum is over all possible partitions $S, R$ of the set $\{1, \cdots, n\}$ such that $S=\left\{s_{1}, \cdots, s_{i}\right\}, \quad s_{1}<s_{2}<\cdots<s_{i}, \quad$ and $R=\left\{r_{1}, r_{2}, \cdots, r_{n-i}\right\}, \quad r_{1}<\cdots<r_{n-i}$; $E(i)=0$ if $i=0, n$ and for $0<i<n, E(i)$ is the image of

$$
\Sigma \operatorname{Tor}\left(A^{s_{1}}, \cdots, A^{s_{i+1}}\right) \otimes A^{r_{1}} \otimes \cdots \otimes A^{r_{n-i-1}}
$$

under the map

$\tau_{h}\left(a^{s_{1}}, \cdots, a^{s_{i+1}}\right) \otimes a^{r_{1}} \otimes \cdots \otimes a^{r_{n}-i-1} \longrightarrow \partial \tau_{h}\left(a^{s_{1}}, \cdots, a^{s_{r+1}}\right) \otimes a^{r_{1}} \otimes \cdots \otimes a^{r_{n-i-1}}$, where

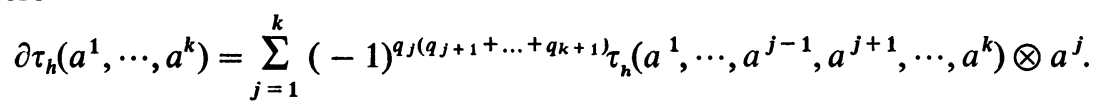

There is an isomorphism

$$
\text { G: } B-\operatorname{Mult}_{i}\left(A^{1}, \cdots, A^{n}\right) \longrightarrow \operatorname{Mult}_{i-1}\left(A^{1}, \cdots, A^{n}\right)
$$

given as follows. If

$$
\tau_{m}\left(a^{s_{1}}, \cdots, a^{s_{t}}\right) \otimes a^{r_{1}} \otimes \cdots \otimes a^{r_{n-t}}
$$

is a generator of $B$-Mult ${ }_{i}\left(A^{1}, \cdots, A^{n}\right)$, then each $a^{s_{k}} \in_{m} A^{s_{k}}(k=1, \cdots, i)$ and $a^{r_{k}}$ can be considered as representing an element of $A^{r_{k}} / m A^{r_{k}}=A_{m}^{r_{k}}(k=1, \cdots,(n-i))$. Thus the element $a^{1} \otimes \cdots \otimes a^{n}$ can be considered as an element of the group:

$$
j_{1} m A_{\left(1-j_{1}\right) m}^{1} \otimes \cdots \otimes_{j_{n} m} A_{\left(1-j_{n}\right) m}^{n},
$$

where $j=\left(j_{1}, \cdots, j_{n}\right)$ is given by:

$$
\begin{array}{ll}
j_{t}=0 & \text { if } t \in\left\{r_{k} \mid 1 \leqq k \leqq n-i\right\} ; \\
j_{t}=1 & \text { if } t \in\left\{s_{k} \mid 1 \leqq k \leqq i\right\} .
\end{array}
$$

We therefore define

$$
\left.G\left[\tau_{m}\left(a^{s_{1}}, \cdots, a^{s_{t}}\right) \otimes a^{r_{1}} \otimes \cdots \otimes a^{r_{n-t}}\right)\right]
$$

to be (the quotient class of) $a^{1} \otimes \cdots \otimes a^{n}$ in the group 


$$
\left\{A_{(j)}^{1}\right\} \otimes \cdots \otimes\left\{A_{(j)}^{n}\right\}
$$

( $j$ determined as above). It is easily verified that $G$ respects the defining relations for the $\tau_{m}\left(a^{s_{1}}, \cdots, a^{s_{t}}\right)$ and is a well-defined homomorphism

$$
G: \sum \operatorname{Tor}\left(A^{s_{1}}, \cdots, A^{s_{1}}\right) \otimes A^{r_{1}} \otimes \cdots \otimes A^{r_{n-1}} \longrightarrow \sum_{j \in J(i)} \bigotimes_{r=1}^{n}\left\{A_{(j)}^{r_{j}}\right\}
$$

(first sum as in the definition of $B$-Mult).

If $a^{1} \otimes \cdots \otimes a^{n}$ is an element of

$$
{ }_{j_{1} m} A_{\left(1-j_{1}\right) m}^{1} \otimes \cdots \otimes_{j_{n} m} A_{\left(1-i_{n}\right) m}^{n} \quad(\text { for } j \in J(i)),
$$

then there is an inverse map for $G$ given by

$$
G^{-1}\left[a^{1} \otimes \cdots \otimes a^{n}\right]=\tau_{m}\left(a^{s_{1}}, \cdots, a^{s_{t}}\right) \otimes a^{r_{1}} \otimes \cdots \otimes a^{r_{n-i}},
$$

where $s_{1}, \cdots, s_{i}$ are those indices $k$ such that $j_{k}=1$ and the $r_{1}, \cdots, r_{n-i}$ are those indices $k$ such that $j_{k}=0$.

One verifies that $G^{-1}$ is well defined and that $G^{-1} G=1=G G^{-1}$. Hence $G$ is an isomorphism. Inspection shows that under the map $G$ the subgroup $E(i)$ is mapped (up to signs on the homogeneous factors) into the subgroup $\tilde{E}(i-1)$. Similarly $G^{-1}$ maps $\tilde{E}(i-1)$ into $E(i)$. Hence $G$ induces an isomorphism of quotients, $G$. But the respective quotients are precisely the groups $B$ - $\operatorname{Mult}_{i}\left(A^{1}, \cdots, A^{n}\right)$ and $\operatorname{Mult}_{i-1}\left(A^{1}, \cdots, A^{i n}\right)$, which are therefore isomorphic.

\section{REFERENCES}

1. M. Bockstein, Splitting in Künneth's formula, Soviet Math. Dokl. 3 (1962), 1267-1270.

2. H. Cartan and S. Eilenberg, Homological algebra, Princeton Univ. Press, Princeton, N. J., 1956.

3. T. Hungerford, Bockstein spectra, Trans. Amer. Math. Soc. (to appear). $51-64$.

4. S. MacLane, Triple torsion products and multiple Künneth formulas, Math. Ann. 140(1960),

5. —, Homology, Springer, Berlin, 1963.

6. M. Bockstein, On the Künneth formula in homological algebra, Dokl. Akad. Nauk SSSR 124 (1959), 1187-1190. (Russian)

7. G. M. Kelly, Observations on the Künneth theorem, Proc. Cambridge Philos. Soc. 59 (1963), 575-587.

8. A. Dold, Zur Homotopietheorie der Kettenkomplexe, Math. Ann. 140 (1960), 278-298.

9. M. Bockstein, A multiple Künneth formula, Izv. Akad. Nauk SSSR. Ser. Mat. 27 (1963), 467-482. (Russian)

10. J. H. C. Whitehead, On simply-connected four-dimensional polyhedra, Comment. Math. Helv. 22 (1949), 48-90.

\section{UNIVERSITY OF WASHINGTON,} SEATtLe, WAShington 